\title{
Novel Members of the Mitogen-Activated Protein Kinase Activator Family in Xenopus laevis

\author{
BEVERLY M. YASHAR, ${ }^{1}$ CLAIR KELLEY, ${ }^{2}$ KAREN YEE, ${ }^{2}$ BEVERLY ERREDE, ${ }^{1}$ \\ AND LEONARD I. ZON ${ }^{2 *}$ \\ and Division of Hematology/Oncology, Children's Hospital, Harvard Medical School, \\ Boston, Massachusetts $02115^{2}$
} \\ Department of Chemistry, University of North Carolina at Chapel Hill, Chapel Hill, North Carolina 27599, ${ }^{1}$
}

Received 29 March 1993/Returned for modification 29 April 1993/Accepted 18 June 1993

\begin{abstract}
Mitogen-activated protein (MAP) kinases comprise an evolutionarily conserved family of proteins that includes at least three vertebrate protein kinases (p42, p44, and p55 MAPK) and five yeast protein kinases (SPK1, MPK1, HOG1, FUS3, and KSS1). Members of this family are activated by a variety of extracellular agents that influence cellular proliferation and difierentiation. In Saccharomyces cerevisiae, there are multiple physiologically distinct MAP kinase activation pathways composed of structurally related kinases. The recently cloned vertebrate MAP kinase activators are structurally related to MAP kinase activators in these yeast pathways. These similarities suggest that homologous kinase cascades are utilized for signal transduction in many, if not all, eukaryotes. We have identified additional members of the MAP kinase activator family in Xenopus laevis by a polymerase chain reaction-based analysis of embryonic cDNAs. One of the clones identified (XMEK2) encodes a unique predicted protein kinase that is similar to the previously reported activator (MAPKK) in X. laevis. XMEK2, a highly expressed maternal mRNA, is developmentally regulated during embryogenesis and expressed in brain and muscle. Expression of XMEK2 in yeast cells suppressed the growth defect associated with loss of the yeast MAP kinase activator homologs, MKK1 and MKK2. Partial sequence of a second cDNA clone (XMEK3) identified yet another potential MAP kinase activator. The pattern of expression of XMEK3 is distinct from that of p42 MAPK and XMEK2. The high degree of amino acid sequence similarity of XMEK2, XMEK3, and MAPKK suggests that these three are related members of an amphibian family of protein kinases involved in the activation of MAP kinase. Discovery of this family suggests that multiple MAP kinase activation pathways similar to those in yeast cells exist in vertebrates.
\end{abstract}

Vertebrate mitogen-activated protein (MAP) kinases (or extracellular-regulated kinases [ERKs]) are signaling molecules common to pathways that regulate proliferation and differentiation in many eukaryotes. Their activation occurs in mitogenic responses, such as epidermal growth-factor (EGF) stimulation of fibroblasts, insulin stimulation of skeletal muscle, and cytokine activation of hematopoietic cells. In addition, MAP kinases are activated in differentiation or nonmitogenic responses, such as oocyte maturation response to progesterone, nerve growth factor (NGF) or bradykinin induction in PC12 cells, catecholamine secretion in response to nicotine in adrenal cells, and response to seizure in the hippocampus (for reviews, see references 5 , 38 , and 52).

Numerous biochemical and developmental studies of the activation of MAP kinase (p42 MAPK) have utilized maturing Xenopus laevis oocytes. Progesterone-induced meiotic maturation of oocytes induces an $\mu$ phase-promoting factor (MPF)-dependent kinase cascade that activates p42 MAPK $(12,16,40)$. In maturing oocytes the upstream activator of MAP kinase (MAP kinase kinase [MAPKK]) is a $45-\mathrm{kDa}$ dual-specificity protein kinase able to phosphorylate p42 MAPK on threonine and tyrosine residues $(32,39)$. $X$. laevis p42 MAPK RNA is expressed in the early oocyte as an abundant maternal message and maintained at a constant level during embryogenesis. At the mid-blastula transition p42 MAPK RNA levels decline (56). Later in development,

\footnotetext{
* Corresponding author.
}

the p42 MAPK and MAPKK proteins can be detected in many tissues of the larval and adult animal $(26,56)$.

While an understanding of the signaling events leading to MAP kinase activation in vertebrates is just beginning to emerge, more is known about signal pathways leading to MAP kinase activation in Saccharomyces cerevisiae. In this yeast, four MAP kinase family members (FUS3, KSS1, MPK1, and HOG1) have been identified $(2,6,9,28)$. These MAP kinase homologs function in three distinct signal transduction pathways (Fig. 1). The most thoroughly defined of these pathways controls the pheromone-induced mating response (for reviews, see references 31 and 48). The protein kinases STE11 and STE7 and the functionally redundant FUS3 and KSS1 kinases are components of this intracellular signal pathway (for a review, see reference 11). Genetic epistasis and biochemical analyses indicate that STE11 kinase acts before the STE7, FUS3, and KSS1 steps in signal transmission $(3,13,50,57)$. Recently, it was demonstrated that STE7 directly activates the MAP kinase homolog, FUS3, by phosphorylation at the predicted Thr and Tyr residues (10).

On the basis of structural homology to STE7, three other $S$. cerevisiae kinases (MKK1, MKK2, and PBS2) are potential MAP kinase activators $(1,22)$. The PBS2 kinase is an upstream activator of the HOG1-MAP kinase homolog, and the functionally redundant MKK1 and MKK2 kinases act in the PKC1-mediated signal transduction pathway upstream of the MPK1-MAP kinase homolog $(2,22)$. Additionally, a STE11-like enzyme (BCK1) has been found to act upstream 


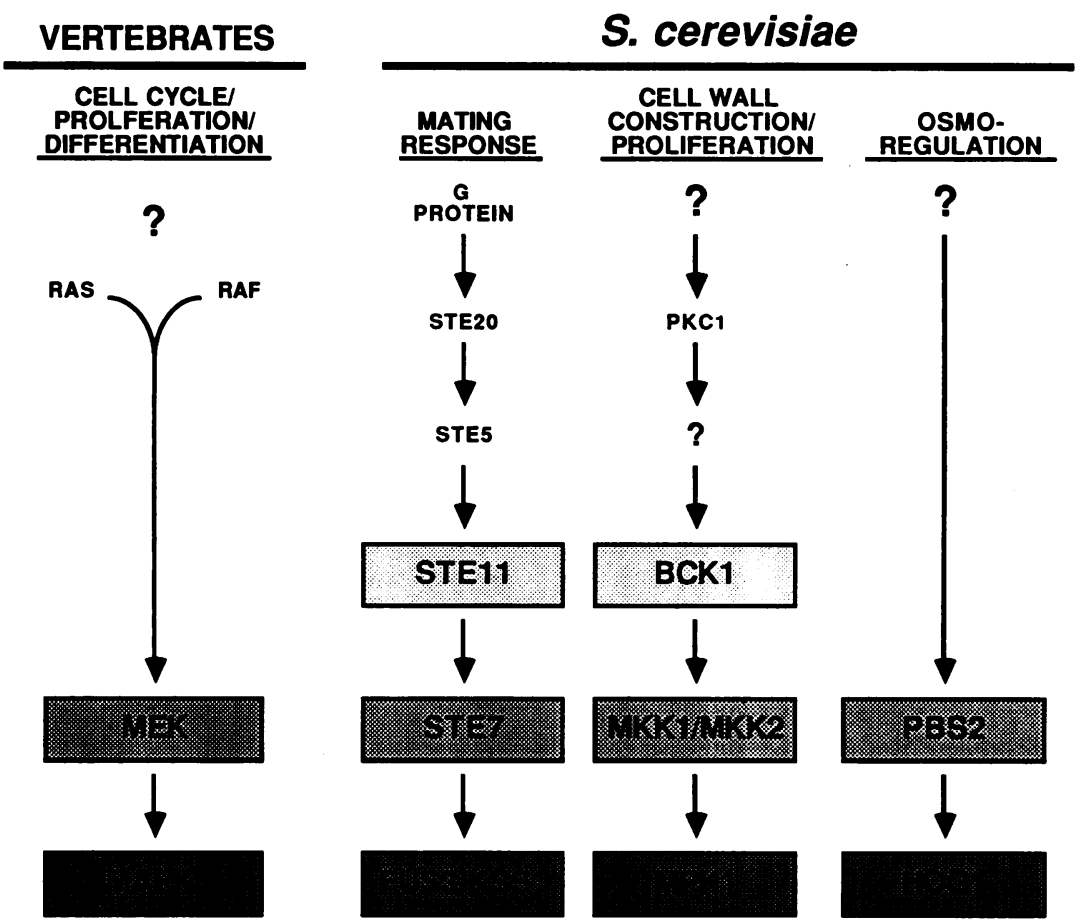

FIG. 1. Comparison of signal transduction pathways of $S$. cerevisiae and vertebrates. Arrows indicate activation or stimulation. Question marks indicate unknown factors active in the signaling pathway. Structurally related kinases are indicated at parallel positions in their respective pathways.

of the MKK1 and MKK2 kinases (29, 42). This striking reiteration suggests that the kinases comprising each pathway function as a common unit, or module, and that this unit has been conserved to mediate different physiological responses in yeast. We anticipate that a similar reiteration of signal transduction modules will function in vertebrates to mediate responses to different extracellular stimuli.

Members of the STE7 protein kinase family have been isolated recently from Xenopus laevis (MAPKK), rats (MAPKK), and mice (MAPK-ERK kinase [MEK]) $(7,26$, 34). Each of these is a $45-\mathrm{kDa}$ dual specificity protein kinase that phosphorylates its substrate, MAP kinase, on the tyrosine and threonine residues necessary for activation $(8,32$, 35). cDNA and predicted protein sequences suggest that the three purified forms of the MAP kinase activators are homologs $(7,27,55)$. The predicted proteins encoded by the two mammalian activators are identical at 383 of 384 residues, while the mammalian and $X$. laevis homologs share $92 \%$ identity.

The catalytic domains of all kinases are characterized by 15 highly conserved residues spread throughout 11 subdomains (17). In both the MAP kinase and MAP kinase activator subfamilies, homologies extend across larger blocks of related amino acids within the catalytic domains. We reasoned that additional members of the MAP kinase activator family could be identified on the basis of these extended regions of homology. We have used a polymerase chain reaction (PCR) analysis with degenerate oligonucleotides to identify novel members of the MAP kinase activator family. Our analysis indicates that in $X$. laevis there are at least three distinct members of the MAP kinase activator family. We have examined the developmental tissue-specific pattern of expression of these novel family members.

\section{MATERIALS AND METHODS}

Embryos and cell lines. $X$. laevis embryos were obtained by standard procedures. The $X$. laevis XTC, XL, and X58 cell lines were obtained from Doug Melton (Harvard University) and the XIF (fibroblast) cell line was obtained from David Shapiro (University of Illinois). The WMPA and PRKT Rana pipiens cell lines were obtained from K. S. Tweedle (Notre Dame).

Yeast strains, genetic procedures, and functional assays. The $S$. cerevisiae strains used in this study have all been previously described. The strain designations and the pertinent genotypes are as follows: 3233-1B, mkk1 $:: L E U 2$

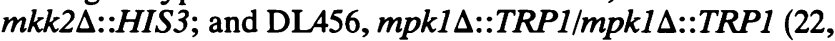
$28,29)$. Unless otherwise specified, we used media and genetic procedures as described in Sherman et al. (46). Yeast strains were transformed by the procedure of Ito et al. (24). Mating assays were performed as described by Sprague (47), sensitivity to high osmolarity medium was assayed as described by Brewster et al. (2), and osmotic suppression of temperature-dependent cell lysis was assayed as described in Irie et al. (22).

PCR. The XMEK2 gene was amplified from $1 \mu$ l of a stage $17 X$. laevis embryo cDNA library $\left(10^{9}\right.$ plaques per $\left.\mathrm{ml}\right)$ with the XMO1 sense and XMO2 antisense degenerate oligonucleotide primers (Table 1). PCR primers were synthesized on an Applied Biosystems Inc. automated oligonucleotide synthesizer, and Taq DNA polymerase was purchased from Perkin-Elmer Cetus. All PCRs described in this paper contained $10 \mathrm{mM}$ Tris- $\mathrm{HCl}(\mathrm{pH} 8.3), 50 \mathrm{mM} \mathrm{KCl}, 1.5 \mathrm{mM}$ $\mathrm{MgCl}_{2}, 0.01 \%$ (wt/vol) gelatin, $200 \mu \mathrm{M}$ all four deoxynucleoside triphosphates, $100 \mathrm{pmol}$ of each primer, $2.5 \mathrm{U}$ of AmpliTaq. Conditions for PCRs performed in a Perkin- 
TABLE 1. Degenerate oligonucleotide primers

\begin{tabular}{|c|c|c|c|}
\hline Name & DNA sequence ${ }^{a}$ & $\begin{array}{l}\text { Amino acid } \\
\text { sequence }\end{array}$ & $\begin{array}{c}\text { Kinase } \\
\text { domain }^{b}\end{array}$ \\
\hline XMO1 & 5'-CGGATCCAYMGNGAYATHAARWSNAARAA-3' & RDIKSKL/N & VIb \\
\hline XMO2 & 5'-GGAATTCCYYTCNGGNGCCATRTA-3' & EPAMY & VIII \\
\hline XMO3 & 5'-CTCGGATCCATHATNCAYMGNGA-3' & II/MHRD & VIb \\
\hline XMO4 & 5'-GGTCTAGAYTCNGGNGCCATRTA-3' & EPAMY & VIII \\
\hline XMO5 & 5'-GGTCTAGAYTCNGGNGNCATRTA-3' & EPSMY & VIII \\
\hline
\end{tabular}

a The underlined nucleotides indicate the restriction enzyme sites added for cloning purposes. Primers XMO1 and XMO3 contain Bam $\mathrm{HI}$ sites, primer XMO2 encodes an EcoRI site, and primers XMO4 and XMO5 encode XbaI sites. Degenerate nucleotide positions are indicated by the following International Union of Biochemistry symbols: $M=A$ or $C ; N=A, C, G$, or $T ; W=T$ or $A ; R=A$ or $G ; H=A, C$, or $T$; $Y=C$ or $T$; and $S=C$ or $G$.

${ }^{b}$ Domain refers to the kinase catalytic subdomains defined by Hanks and Quinn (16).

Elmer thermocycler were $1 \mathrm{~min}$ at $94^{\circ} \mathrm{C}, 1 \mathrm{~min}$ at $37^{\circ} \mathrm{C}$, and $3 \mathrm{~min}$ at $63^{\circ} \mathrm{C}$ for 30 cycles, with a final 7 -min extension at $63^{\circ} \mathrm{C}$. The resultant PCR products were digested with EcoRI and BamHI enzymes, purified by standard methods, and ligated into the Bluescript II SK- vector. DNA was prepared from recombinant clones, and the inserts were sequenced by the dideoxy method (Sequenase version 2.0, DNA Sequencing Kit; U.S. Biochemical Corp.). The clone containing the XMEK2 PCR fragment was designated pXSTE7.

PCR-based analysis of individual $X$. laevis stage 17 cDNA clones utilized the XMO3 sense and XMO4-XMO5 antisense degenerate oligonucleotide primers (Table 1). Equimolar amounts of all three primers were included in the PCRs as described above. The PCR product of the appropriate size was gel purified, digested with BamHI and XbaI enzymes, and cloned into pUC118. Clones were sequenced by the dideoxy chain termination method with Klenow polymerase (45). All enzymes were purchased from New England Biolabs, Bethesda Research Laboratories, United States Biochemicals, Stratagene, or Promega.

Analysis of cDNA libraries. A stage $17 \mathrm{X}$. laevis cDNA library (Doug Melton, Harvard University) was screened by plaque hybridization (44). The XMEK2 insert was removed from pXSTE7 by digestion with HindIII and XbaI, gel purified, and labeled by nick translation with Escherichia coli DNA PolI to generate a probe (44). Hybridizations were carried out at $65^{\circ} \mathrm{C}$ in $6 \times \mathrm{SSC}(20 \times \mathrm{SSC}$ is $3 \mathrm{M} \mathrm{NaCl}$ plus 0.3 $M$ sodium citrate) $-0.05 \times$ BLOTTO ( $1 \times$ BLOTTO is $5 \%$ nonfat dried milk plus $0.02 \%$ sodium azide) from 1 to $3 \times$ $\cot _{1 / 2}$. Filters were washed at high stringency through a decreasing series of SSC washes $(2 \times, 1 \times, 0.5 \times$, and $0.2 \times)$ and exposed to Kodak XAR film overnight at $-70^{\circ} \mathrm{C}$ with an intensifying screen. Plaques that yielded a positive signal in the initial screen were plaque purified and screened by plaque hybridization a second time as described above (44).

Recombinant lambda plaques that reproducibly hybridized to the XMEK2 fragment of pXSTE7 were plaque purified (44). These cDNA clones were categorized into subfamilies by a PCR-based analysis with the degenerate oligonucleotide primers XMO3, XMO4, and XMO5 (Table 1). DNA was prepared from plate lysates of individual plaques of the clones selected for subsequent analyses and digested at the EcoRI sites marking the endpoints of cDNA insertion. cDNA inserts were gel purified and cloned into the EcoRI site of pUC118. Clones were categorized into subfamilies by restriction maps. The clone with the strongest reproducible pattern of hybridization and the largest cDNA insert was designated pNC353 and used for subsequent analyses.

Two $X$. laevis oocyte cDNA libraries and an $X$. laevis stage 45 cDNA library were screened with pNC353. The oocyte and stage $45 X$. laevis embryo cDNA libraries were provided by D. DeSimione (University of Virginia). Positively hybridizing clones were categorized into subfamilies on the basis of the sequences at the $5^{\prime}$ and $3^{\prime}$ ends of the cDNA inserts, restriction maps, and a PCR-based analysis of internal regions.

DNA cloning and sequence determinations of XMEK2. Isolates of XMEK2 cloned into the EcoRI site of pUC118 in both orientations (pNC353 and pNC354) were used to generate banks of subclones by progressive unidirectional deletion with exonuclease III (19). Subclones in these banks were sequenced by the dideoxy-chain termination method with Klenow polymerase, and the DNA sequence data were assembled by using DBSYSTEM programs on a VAX-11/780 computer $(45,49)$.

DNA and protein alignments were performed with the Genetics Computer Group programs (14) on a VAX-11/780 computer.

Site-directed mutagenesis of XMEK2. A lysine-to-arginine mutation was created in the presumptive ATP-binding site of XMEK2 by using an overlapping PCR technique with the PCR primers XMEKMUT (XMEKMUT, 5' AGATTATG GCTGTAAGGAGGATTCGCTCCAC 3'), the complementary oligonucleotide XMEK2MUTR, and the T3 and T7 primers (20). The template in these PCR was the XMEK2 cDNA cloned into the EcoRI site of the pBluescript II KSplasmid (Stratagene). This mutagenesis generates an A-to-G transposition at position 534 in the XMEK2 cDNA (Fig. 3). The final PCR product was digested with the restriction endonuclease EcoRI, gel purified, and cloned into the EcoRI site of pBluescript II KS-. The mutation at amino acid 160 was confirmed by sequencing, and the resulting plasmid was called pXMEK2-R160. The position of the mutated amino acid is numbered from the first methionine in the XMEK2 open reading frame (see Fig. 3).

Yeast expression plasmids. $X M E K 2$ and $X M E K 2-R 160$ were cloned into a yeast CEN3 TRP1 ARS1 vector (pNC161) that contains the triosephosphate isomerase (TPI1) promoter (41). This construct allows for constitutive expression of either the XMEK2 wild-type or the lysine-to-arginine XMEK2 mutant protein in yeast cells. A 1.7-kb fragment that contains the entire predicted open reading frame of the protein was removed from either pNC353 or pXMEK2-R160 by digestion with NarI enzyme (position 1715; see Fig. 3), end-repairing, and then digestion with EcoRI enzyme. The TPI1 promoter was isolated as a $900-b p$ BglII-EcoRI fragment from pGA1484 (G. Ammerer, Vienna, Austria). In a single ligation step, the two fragments were joined at their $E c o$ RI ends and inserted into the pNC161 vector that had been linearized by digestion with BamHI enzyme and end- 


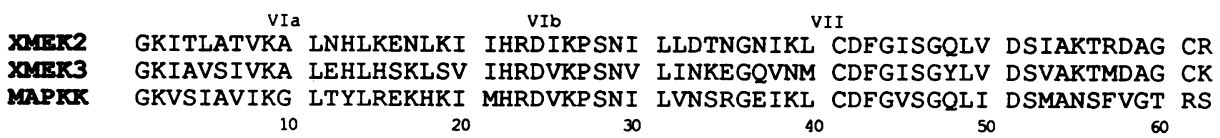

FIG. 2. The MEK family of $X$. laevis. Alignment of the deduced protein sequences in domains VI and VII of XMEK2, XMEK3, and MAPKK of $X$. laevis (27). Roman numerals refer to the conserved kinase domains defined by Hanks and Quinn (17).

repaired PstI. The desired recombinant clones were identified by restriction analysis and given the designation pNC341 or pNC341-R160.

The TPI1-XMEK2 allele was also cloned into a CEN4 ARS1 URA3 vector. A 2.5-kb TPI1-XMEK2 fragment was removed from pNC341 by digestion with the restriction endonucleases KpnI and HindIII and ligated into the complementary sites in the vector YCplac33 (15). The desired recombinant clone was identified by restriction analysis and given the designation pNC373. Standard recombinant DNA procedures were used for all plasmid constructions and preparations (44). The vectors pNC161 and YCplac33 were used as negative controls in the yeast expression studies (15, 41).

Plasmids containing a 2.7-kb SalI-PstI fragment encoding the $M K K 1$ gene and promoter in either YCplac112 (p112-32) or YCplac195 (p195-32) were provided by K. Matsumoto (22). The YEp13-MPK1 plasmid containing the MPK1 gene and its promoter was provided by D. E. Levin (28).

Embryo RNA preparation. $X$. laevis embryos were lysed in proteinase $\mathrm{K}(200 \mu \mathrm{g} / \mathrm{ml})$ in $50 \mathrm{mM}$ Tris- $\mathrm{HCl}(\mathrm{pH} \mathrm{7.5),} 50$ $\mathrm{mM} \mathrm{NaCl}, 5 \mathrm{mM}$ EDTA, and $0.5 \%$ sodium dodecyl sulfate and incubated for $30 \mathrm{~min}$ to $1 \mathrm{~h}$ at $37^{\circ} \mathrm{C} \mathrm{(53).} \mathrm{The} \mathrm{lysate} \mathrm{was}$ extracted with phenol-chloroform twice and ethanol precipitated. The precipitate was resuspended in diethylpyrocarbonate (DEPC)-treated water, an equal volume of $8 \mathrm{M} \mathrm{LiCl}$ was added, and the RNA was precipitated overnight at $-20^{\circ} \mathrm{C}$. The RNA was collected by centrifugation, washed with $70 \%$ ethanol, resuspended in DEPC-treated water, and stored at $-80^{\circ} \mathrm{C}$.

RNA preparation from tissue culture cells. Cells were grown in L-15 medium Leibovitz (Sigma) in 10\% fetal bovine serum with penicillin $(50 \mathrm{U} / \mathrm{ml})$ and streptomycin sulfate $(50$ $\mu \mathrm{g} / \mathrm{ml})$ at $25^{\circ} \mathrm{C}$. Cells were washed twice in saline and lysed directly on the plate with solution D, and RNA was extracted from whole cells by the single-step guanidinium isothiocyanate extraction with the following modification: after the initial lysis and extraction with phenol-chloroform, a second phenol-chloroform extraction was performed before ethanol precipitation (4). Samples were stored at $-80^{\circ} \mathrm{C}$.

Northern blot analysis. Total cellular RNA $(10 \mu \mathrm{g})$ was loaded on a formaldehyde-1\% agarose gel (44). Standard methods were used for transfer to a Magnagraph membrane and subsequent hybridization with a randomly labeled probe (44). After hybridization, each blot was stripped of radioactivity and reprobed with an ornithine decarboxylase PCR fragment as an internal blotting control (23).

Whole embryo in situ analysis. Whole embryo in situ analysis was performed on staged albino embryos as described by Hemmati-Brivanlou et al. (18). XMEK2 antisense- and control sense-strand probes were generated from linearized pNC353 plasmids. Probes were synthesized by incorporating digoxigenin-11 UTP by using bacterial RNA polymerases (T3 or T7) (18). The hybridization signal was detected by secondary antibody against digoxigenin coupled to alkaline phosphatase, followed by development with nitroblue tetrazolium - 5 - bromo -chloro - 3 - indolyl-phosphate toluidinium (NBT-BCIP) (Promega). For staining analysis, whole embryos were cleared with a 2:1 solution of benzyl benzoate-benzyl alcohol (BB-BA) (18).

Nucleotide sequence accession number. The EMBL accession number for XMEK2 is Z22736.

\section{RESULTS}

Identification of members of the XMEK family. A cDNA library from $X$. laevis embryos (Nieuwkoop stage 17) was screened by PCR-based amplification $(36,43)$. DNA sequence analysis of cloned amplification products identified a PCR product with an open reading frame of 50 amino acid residues. The predicted protein encoded by this fragment showed strong similarity to the members of the STE7 family of protein kinases. Members of this family include STE7, BYR1, MKK1, MKK2, PBS2, and WIS1 (1, 22, 33, 51, 54). The predicted amino acid sequence of the PCR product mapped to kinase catalytic subdomains VIB, VII, and VIII (17). To identify $X$. laevis cDNA clones containing this sequence, the PCR-amplified fragment was used as a probe in a plaque hybridization screen of a stage $17 \mathrm{X}$. laevis embryonic cDNA library. Ten positive plaques were identified in a screen of $4 \times 10^{6}$ PFU from this cDNA library.

A PCR-based amplification was utilized to determine whether the sequences of the 10 positive clones were similar or different (see Materials and Methods). This analysis allowed us to group the clones into three categories. Three of the cDNA clones contained regions that encoded 50 amino acids identical to those in the original amplified fragment. Restriction analyses of the three clones suggest that they represent different lengths of cDNAs derived from the same gene. We have named the gene coding for these cDNA clones Xenopus MAPK-ERK kinase 2 (XMEK2) (Fig. 2). Two of the 10 cDNA clones contained identical DNA fragments encoding a predicted protein fragment that was related to XMEK2. The single open reading frame in these clones encodes a unique protein kinase with $63 \%$ identity to XMEK2 (Fig. 2). The gene encoding these cDNAs was named Xenopus MAP-ERK kinase 3 (XMEK3). The $\mathrm{XMEK} 2$ and $\mathrm{XMEK} 3$ predicted proteins are distinct from the biochemically identified $X$. laevis MAP kinase activator protein (27). Within subdomains VI to VIII, XMEK2 is $62 \%$ identical and XMEK3 is $46 \%$ identical to the $X$. laevis MAP kinase activator protein (Fig. 2). On the basis of the structural similarity, XMEK2 and XMEK3 represent two new members of the MAP kinase activator family in $X$. laevis.

The remaining five clones were weak positives that produced anomalous results under the same PCR conditions: two of these cDNA clones did not produce a PCR product. In the remaining three, a fragment was amplified from the lambda exonuclease gene (data not shown).

Primary structure of XMEK2. We sequenced the XMEK2 clone with the largest cDNA insert $(1,807$ nucleotides) on both strands (Fig. 3). The DNA sequence of the clone encodes a single open reading frame starting at position 2 


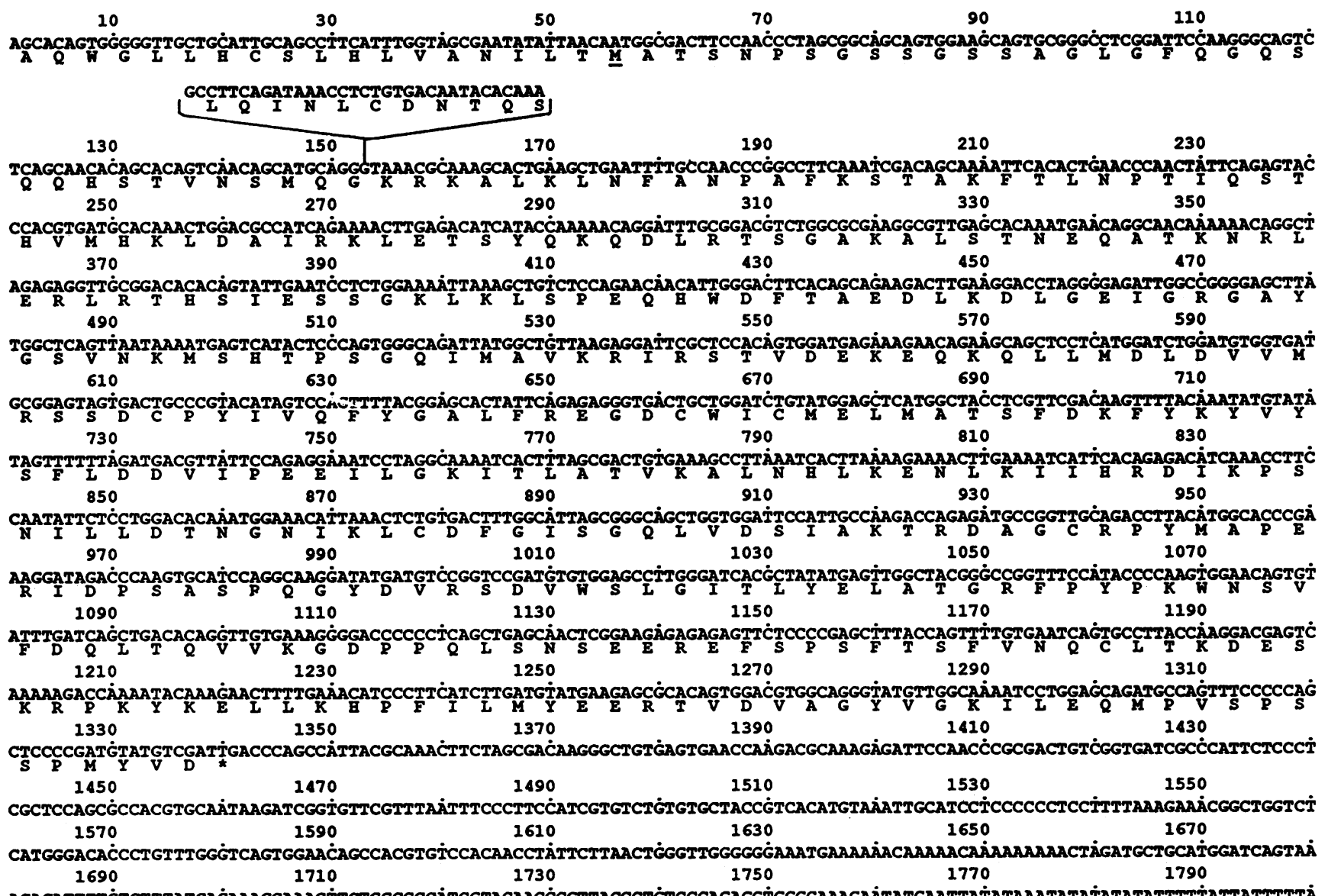

AAAAAA

FIG. 3. Nucleotide and predicted protein sequences of XMEK2. The nucleotide sequence of the coding strand is shown. The sequenced insertion found for XMEK2 variant clones is indicated at position 153 (see Results [primary structure of XHEKZ]). The first methionine in the predicted protein sequence is underlined. ', every 10 th position in the nucleotide sequence; $*$, termination codon.

and terminating with a TGA translation termination codon at position 1337.

Sequence analyses of independently isolated XMEK2 cDNAs from oocyte and stage 45 cDNA libraries suggest that alternately processed forms of XMEK2 are expressed. In these cDNA libraries, the primary structure of the majority of XMEK2 transcripts is identical to that of the original stage 17 cDNA (data not shown). Surprisingly, a variant was identified in the oocyte mRNA population that contains a 33-bp insertion after nucleotide 153. This insertion adds 11 amino acids to the XMEK2 protein predicted by the original cDNA clone (Fig. 3).

The deduced protein sequence of the XMEK2 cDNA clone contains all the conserved signature motifs characteristic of protein kinases (17) (Fig. 3). Searches of current protein data bases by using the FASTA algorithm (SwissProt release no. 21) revealed that the sequence of this predicted $X$. laevis protein kinase is distinct from any listed sequences (37). However, consistent with the homologies noted in the small PCR fragment, the entire sequence shows a high degree of relatedness to six yeast protein kinases (STE7, PBS2, BYR1, MKK1, MKK2, and WIS1), the MAP kinase activator from $X$. laevis (MAPKK), and two mammalian protein kinases (MEK and MAPKK) (Fig. 4) (1, 7, 22, 27, 33, $51,54,55)$. Values for identity (similarity) of aligned sequences by a BestFit analysis ranged from 34 to $47 \%$ identity and 44 to $67 \%$ similarity. Conservation of the amino acid sequence was most striking within the catalytic domains of these proteins (Fig. 4). Values for the percent identity in an alignment of the XMEK2 catalytic domain with the other MAP kinase activator family members ranged from 39 to $51 \%$, while the identities within the regulatory domains were 17 to $25 \%$ (Fig. 5). The only striking similarity within the regulatory domains of the MAP kinase activators is the high serine content near the $\mathrm{N}$ terminus. In XMEK2, 7 of 20 amino acid residues downstream from the first methionine in the open reading frame are serine residues (Fig. 2).

XMEK2 provides MAP kinase activator function in $S$. cerevisiae. To test whether the structural similarity of XMEK2 to members of the MAP kinase activator family has functional significance, we asked whether expression of XMEK2 would suppress the various response defects associated with loss of different MAP kinase activators in $S$. cerevisiae (Fig. 5). We first tested XMEK2 for the ability to provide MKK1 and MKK2 function in the PKC1-mediated signal pathway (Fig. 1). In this pathway, signal transmission is dependent on an ordered kinase cascade in which the BCK1 gene product functions before MKK1 and MKK2 and the MPK1 gene product functions after MKK1 and MKK2 $(22,28,29)$.

A strain with deletions of the $M K K 1$ and $M K K 2$ genes was transformed with either a plasmid that allows for constitutive 


\begin{tabular}{|c|c|c|c|c|c|c|c|c|c|c|}
\hline & 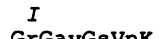 & & II & & $I I I$ & IV & & V & & \\
\hline $\begin{array}{l}\text { XMEK2 } \\
\text { MAPKK }\end{array}$ & $\begin{array}{l}\text { GrGayGsVnK } \\
\text { GAGNGGVVfK }\end{array}$ & $\begin{array}{l}\text { msHtPsgqIM } \\
\text { VsHKPTSIIM }\end{array}$ & $\begin{array}{l}\text { AvKrIRstV. } \\
\text { ARKIIhLEI }\end{array}$ & DekeQkQllm & DLdVVMrssd & CPYIVqFYGA & LF . . r rEGDC & WICMEIM. at & SFDKfyKY & \\
\hline & GAGNGGVVfK & VsHKPsglvM & ARKII HLEI. & kPAI rNQIIR & ELq. VLHeCN & SPYIVGFYGA & $\begin{array}{l}\text { FY.... SDGE } 1 \\
\text { FY. SDGEI }\end{array}$ & S ICMEhMDGG & $\begin{array}{l}\text { SLDqVIK..K } \\
\text { SLDqVIK ..K }\end{array}$ & \\
\hline & GkGNYGVVYK & lHqPTgVtM & AlKeIRLsl. & EeAt fNQIIm & ELd. ILHkav & SPYIVAFYGA & VEGSV & FICMEYMDaG & SMDKlya. q & \\
\hline & ANYGnVSK & IHKPTnVIM & At KeVRLEl & DeAkfrQIlm & ELe.VLHkCN & YIVdFYGA & . IEGaV & YMCMEYMDGG & SIDKIyd..e & . \\
\hline BYR1 & GeGNGGaVsI & $\mathrm{Hr}$. .nIfM & KtVyvgs & DsklQkQIlR & ELg. VLHhCr & YIVGFYGA & .yknnI & SICMEYMDCG & SLDaIİ & \\
\hline STE7 & GAGNsGtVvK & LHvPdskIv & AkKt IpvEqn & nstIINQlvR & ELsIVknvkp & henIItFYGA & YYnqhInnEI & iIIMEYsDCG & SLDKI & $r \pm$ \\
\hline $\begin{array}{l}\text { MKK1 } \\
\text { Cons }\end{array}$ & GeGaGGsVsK & IKngsk If & $1 \mathrm{KvInt} \ln \mathrm{t}$ & DpeyQkQIfR & ELq.fnrsfq & YIVIYYG. & mFtddenss I & YI aMEYMgGr & SLDaIyKnll & e. \\
\hline & $G-G$ & 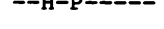 & $5-----2$ & $-0---$ & $=-$ & IV & & D-- & -D- -8 & \\
\hline
\end{tabular}

\begin{tabular}{|c|c|c|c|c|c|c|c|c|c|c|}
\hline \multirow{4}{*}{$\begin{array}{l}\text { MEK2 } \\
\text { MAPKK } \\
\text { MEK }\end{array}$} & \multicolumn{3}{|c|}{ VIA } & \multicolumn{2}{|l|}{$V I B$} & $V I I$ & \multicolumn{4}{|c|}{$V I I I$} \\
\hline & 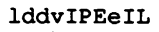 & GKItlAtVKa & LnhLKEnIKI & IHRDIKPSNI & LldtN.GnIK & LCDFGISGQL & IdSiAKTrda & OYMaPE & Idp & Yo \\
\hline & PEkIL & VIKG & LtYLrEKHKI & mHRDVKPSNI & jeIK & LCDFGV & SF.V & גירות & & \\
\hline & GIL & KG & I & SNI & & $\mathrm{LC}$ & F.V & PER & & IW \\
\hline Sara & $L$ & qG & I & IH & L & $\bar{L}$ & n.I & R & & $\mathbf{W}$ \\
\hline $\mathrm{DSc}$ & $\mathrm{qL}$ & & II & NI & & $\mathrm{LCD}$ & n. I & $\mathbf{R}$ & & IW \\
\hline & $L$ & KG & $\operatorname{lhI}$ & NV & & LC & & ER & & IW \\
\hline$E$ & $t i$ & $\operatorname{lng}$ & yKI & IHI & IK & $\mathrm{kL}$ & F.V & $R$ & & VW \\
\hline & rgc & $\operatorname{lrg}$ & YLHEK.KV & IHRDIKPqNI & iqvK & GVSGea & VnslatTF.t & CsfyMaPFR & & VWw \\
\hline & $----E--L$ & & $\mathrm{~L}--\mathrm{L}----\mathrm{I}$ & & $-K$ & SG-L & $S-A-$ & & & \\
\hline
\end{tabular}

\begin{tabular}{|c|c|c|c|c|c|c|c|c|c|c|}
\hline & $I X$ & & & & & & & $x$ & & \\
\hline $\begin{array}{l}\text { XMEK2 } \\
\text { MAPKK }\end{array}$ & $\begin{array}{l}\text { SLGit lYELA } \\
\text { SMGLSIVEMA }\end{array}$ & $\begin{array}{l}\text { TGRFPYPkwn } \\
\text { iGRYP i PPPd }\end{array}$ & akELELiFGc & sVE $\ldots \ldots \ldots$ & $\ddot{1} \ldots \ldots \ldots$ & isSYGó & ...sVFDqLt & qVV? & Lsns & PsFtsFVNGC \\
\hline EK & SMGLSIVEMA & VGRYP IPPDd & akELELIFGC & qVEg & $R$ & & & $\begin{array}{l}\text { P.k } \\
\text { P.k }\end{array}$ & $\begin{array}{l}\text { VFg } \\
\text { VFS }\end{array}$ & $\begin{array}{l}\text { VNKC } \\
\text { VNKC }\end{array}$ \\
\hline NIS & MA & $1 \mathrm{G}$ & & $\cdots$ & $\ldots \ldots$ & - & $\ldots$ & a & $s$ & $\mathrm{P}$ \\
\hline PBS & I IEMA & 1GRYPYPPEt & & & & & Ls & P.r & LPSD & Vsic \\
\hline $3 \mathbf{Y}$ & SLGISIIELA & TqeLP & & $\ldots$ & $\ldots \ldots \ldots w$ & $\mathrm{sn}$ & dsic & P.r & Fp & $\mathrm{VdaC}$ \\
\hline$T$ & SLGLMI IELV & TGeFPlgghn & & & & & $\mathrm{Lq}$ & P.r & YS & IrC \\
\hline IKK1 & LtIIEvA & KFPCSSEk & & & & & aniApEELLM & IltftPelk & pEsniIWS & PsFks \\
\hline & $-E-A$ & & & & & & & ?- & & 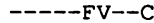 \\
\hline
\end{tabular}

\begin{tabular}{lc} 
& \multicolumn{1}{c}{$X I$} \\
XMEK2 & LtKdeskR \\
MAPKK & LVKNPaER \\
MEK & LIKNPaER \\
WIS1 & LnKNPsIR \\
PBS2 & LqKiPe.R \\
BYR1 & LhKdPt IR \\
STE7 & CIKNerER \\
MKK1 & LKKds IER \\
Consensus & -- K----R
\end{tabular}

FIG. 4. Alignment of the deduced protein sequences of protein kinases in the STE7 kinase family. Uppercase letters designate amino acid residues in the aligned sequences that were either identical or conservative substitutions. Lowercase symbols indicate amino acid residues that were not conserved. The consensus sequence contains residues that were identical in six of the seven aligned sequences. Residues conserved in all kinases are indicated by boldface type in the consensus sequence (17). Roman numerals above the sequences refer to subdomains of the protein kinase catalytic domains described by Hanks and Quinn (17). The sequences are those for XMEK2 (Fig. 3), MAPKK (27), MEK (7), WIS1 (54), PBS2 (1), BYR1 (33), STE7 (51), and MKK1 (22).

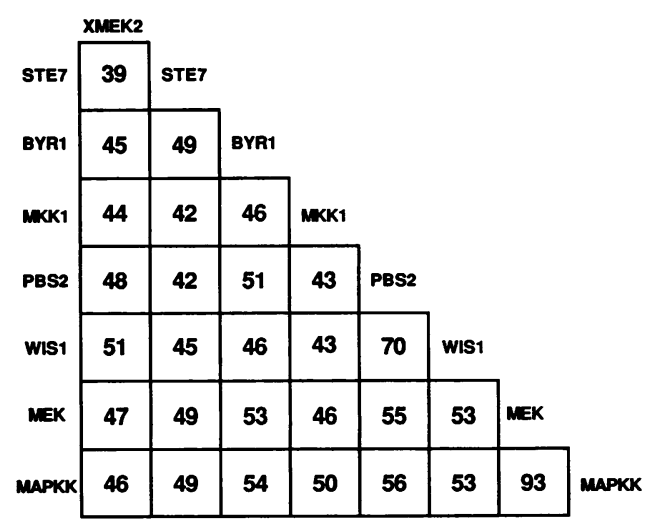

FIG. 5. Percent identities for the catalytic domains of the STE7 protein kinase family. Sequence comparisons were limited to the region bounded by the conserved GXGXXG motif of subdomain I and the conserved $R$ of subdomain XI (17). The percentage of identical amino acids was calculated for each pair by using the Genetics Computer Group BestFit program to maximize the number of matches. The sequences are the same as those listed in Fig. 4. expression of wild-type XMEK2 (pNC341) or a substitution derivative, XMEK2-R160 (pNC341-R160). XMEK2-R160 contains a lysine-to-arginine substitution at the presumptive catalytic lysine in subdomain II of XMEK2 (Fig. 3). To provide negative and positive controls for the functional assay, the same strain was transformed with vector (pNC161) or a plasmid that overexpresses MKK1 (p112MKK1).

Loss of MKK1 and MKK2 results in a temperaturedependent deficiency in cell wall construction that causes cell lysis at the restrictive temperature $\left(37^{\circ} \mathrm{C}\right)$ in the absence of osmotic stabilizers (Fig. 6A, vector) (22). Expression of XMEK2 suppressed the defect associated with loss of MKK1 and MKK2 and allowed cells to form colonies at the restrictive temperature $\left(37^{\circ} \mathrm{C}\right)$ without osmotic stabilizers (Fig. 6A, XMEK2). The extent of suppression by XMEK2 is at least equivalent to the level of complementation by MKK1 (Fig. 6A, MKK1) (22). Suppression of the temperaturedependent cell lysis defect is dependent on XMEK2 kinase function, since $m k k 1 \Delta m k k 2 \Delta$ cells transformed with XMEK2-R160 did not grow at the restrictive temperature in the absence of osmotic stabilizers (Fig. 6A, XMEK2-R160).

The above result does not distinguish whether suppression of MKK1 and MKK2 by XMEK2 is mediated by the downstream MAP kinase (MPK1) or occurs by inappropri- 

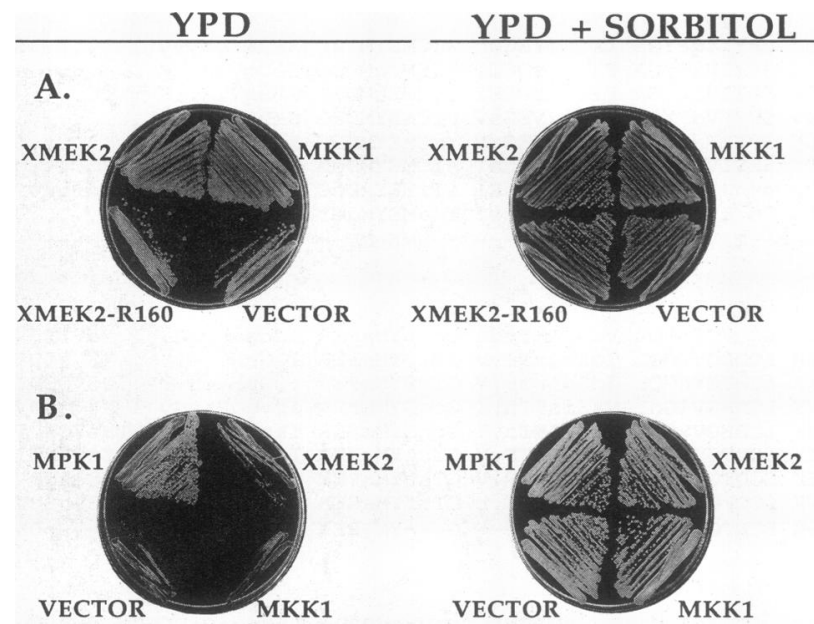

FIG. 6. Expression of XMEK2 suppresses the temperature-sensitive growth defect of a $m k k 1 \Delta m k k 2 \Delta$ strain. (A) Strain 3233-1B $(m k k 1 \Delta / m k k 2 \Delta)$ was transformed with plasmids containing the indicated genes (XMEK2, pNC341; MKK1, p112-32; XMEK2-R160, pNC341-R160; and vector, pNC161), and the transformants were streaked onto YPD medium alone or supplemented with $1 \mathrm{M}$ sorbitol and incubated at $37^{\circ} \mathrm{C}$ for 2 days. (B) Strain DL456 (mpkl $1 \Delta$ ) was transformed with plasmids containing the indicated genes $(X M E K 2$, pNC374; $M K K 1$, p195-32; MPK1, YEp13-MPK1; and vector, YCplac33) and treated as described for panel $A$.

ately activating targets of the pathway. To address this issue we asked whether expression of XMEK2 was able to suppress the defect associated with loss of MPK1. A strain with a deletion of the MPK1 gene was transformed with plasmids that express XMEK2 (pNC341) or MKK1 (p195-32). As controls the same strain was transformed with vector (YCplac33) or MPK1 (YEp13-MPK1). Transformants were tested for the ability to grow at the restrictive temperature $\left(37^{\circ} \mathrm{C}\right)$ in the absence of osmotic stabilizers. As expected, the defect associated with the loss of MPK1 was complemented by its expression (Fig. 6B, MPK1) (28). Expression of XMEK2 or MKK1 did not suppress the defect (Fig. 6B, XMEK2 and MKK1) (28). These results are consistent with $\mathrm{XMEK} 2$ function in the PKC1-mediated pathway requiring MAP kinase activity. They strengthen the argument that XMEK2 is a MAP kinase activator.

In contrast to the results with the PKC 1-mediated pathway, XMEK2 expression did not suppress the response defects caused by loss of the STE7 and PBS2 MAP kinase activators. These two enzymes function in the mating and osmoregulation signal pathways, respectively. Strains with a deletion of STE7 (E929-6C-18) expressing either XMEK2 (pNC341) or vector (pNC161) did not mate with an appropriate tester strain and did not activate transcription of a pheromone-inducible reporter gene (Fus1-LacZ) (data not shown). Strains with a disruption of $P B S 2$ (GBH38) expressing either XMEK2 (pNC341) or vector (pNC161) did not grow on medium supplemented with $0.9 \mathrm{M} \mathrm{NaCl}$ (data not shown). Therefore, XMEK2 provides MAP kinase activator function in only one of the yeast signal pathways. This discrimination could reflect an isotype specificity for the PKC1 pathway. Alternatively, XMEK2 may function in the three yeast pathways, but each pathway may have a different signal threshold for response.

Temporal patterns of XMEK 2 and XMEK3 expression. To examine the developmental expression of XMEK2, we per-

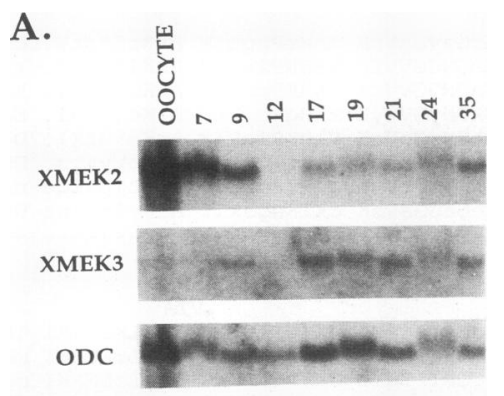

B.

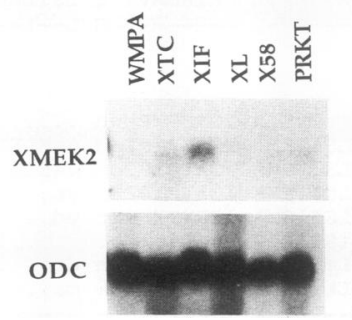

FIG. 7. Northern blot analysis of XMEK2 and XMEK3 expression. Equal amounts of total RNA $(10 \mu \mathrm{g})$ from whole fertilized $X$. laevis embryos at the indicated stages of development (A) or from the indicated frog cell lines (B) were analyzed by Northern blots. Blots were hybridized to either a radiolabeled XMEK2, XMEK3, or ornithine decarboxylase (ODC) probe as indicated. (A) The same blot was hybridized to each of the three probes. Following hybridization to XMEK2, the blot was stripped and rehybridized to the XMEK 3 and ornithine decarboxylase probes. (B) The same blot was hybridized to the XMEK2 and ornithine decarboxylase probes.

formed a Northern blot analysis of RNA derived from different stages of $X$. laevis embryonic development. A single 2.7-kb transcript was identified (Fig. 7A). XMEK2 is expressed as a maternal RNA, which decays during the mid-blastula transition, as evidenced by decreasing levels of the XMEK2 transcript at stage 9. XMEK2 mRNA is expressed as a zygotic transcript by early neurula, stage 17 (Fig. 7A). The level of XMEK2 mRNA expression was examined in a number of $X$. laevis cell lines (XTC, XIF, XL, and X58) and $R$. pipiens cell lines (Fig. 7B). XMEK2 expression was detected in all cell lines and was highest in the XIF fibroblast cell line.

The XMEK2 transcript is at least $900 \mathrm{bp}$ larger than the cDNA insert isolated from the $X$. laevis stage 17 library. On the basis of the sequences of a number of independently isolated XMEK2 cDNAs, we believe that most of this size discrepancy can be accounted for by mispriming at an adenine-rich internal region in the XMEK2 message (Fig. 3). We identified multiple XMEK2 cDNA clones with 2.2-kb inserts that were identical to the sequenced cDNA at the $5^{\prime}$ end but contained characteristic poly(A) tails at their $3^{\prime}$ ends (data not shown). Thus, the $3^{\prime}$ end of the sequenced XMEK2 cDNA clone is probably not the true terminus but a truncation. The fact that the reading frame at the $5^{\prime}$ end of the XMEK2 cDNA is open to the end of the insert raises the possibility that sequences are missing from the $5^{\prime}$ end of the XMEK2 cDNA clone. Although the predicted protein sequence may be incomplete, this possibility does not limit the analyses or conclusions made in this study.

The XMEK3 RNA transcript is $3.5 \mathrm{~kb}$ and its expression 

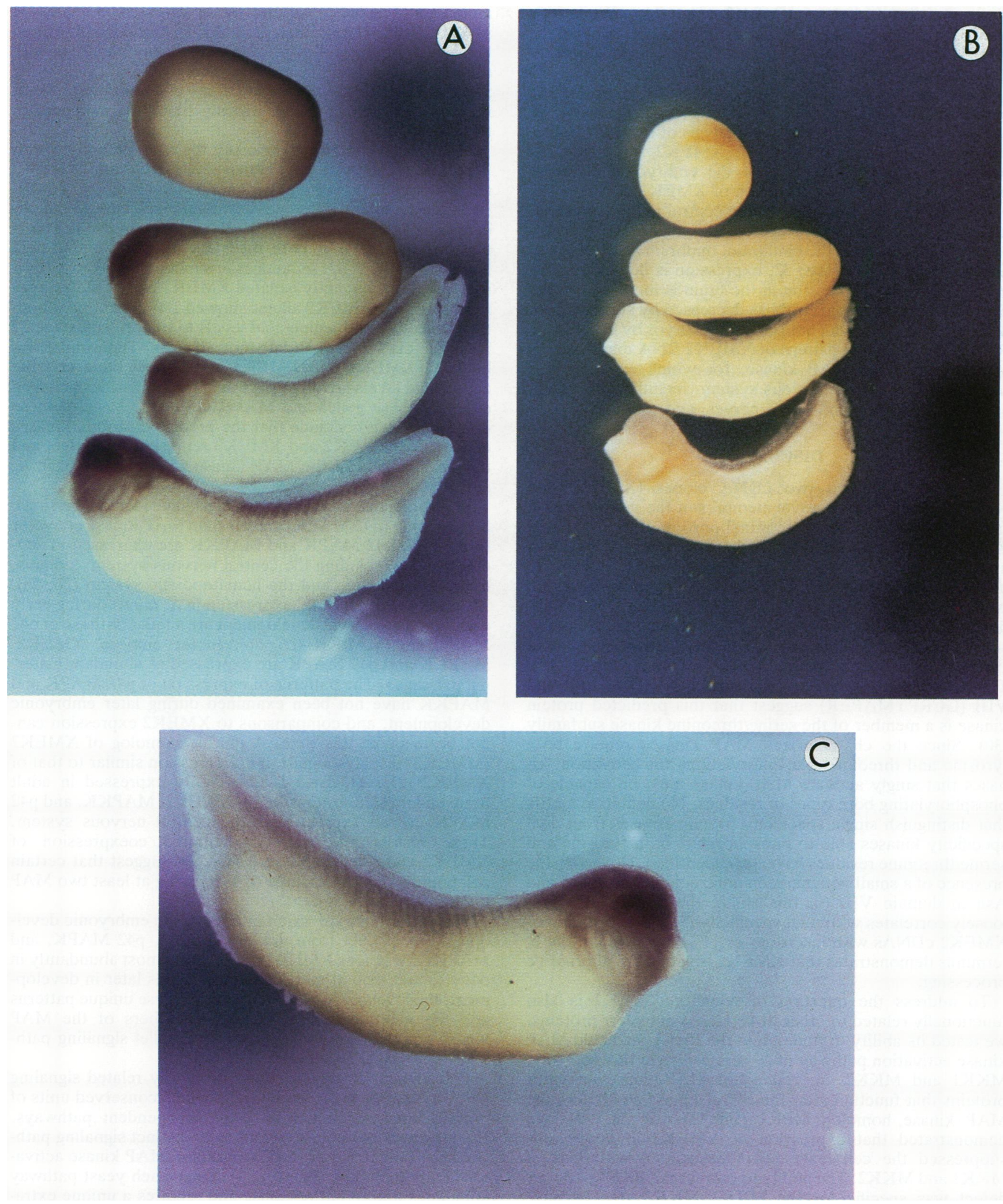

FIG. 8. XMEK2 expression during embryogenesis. Embryos were analyzed by whole mount in situ hybridization to XMEK2 RNA. Shown are $X$. laevis embryos hybridized with either XMEK2 antisense (A and C) or sense (B) strand RNA probes. (A and B) The stages of embryonic development from top to bottom are 17, 24, 32, and 37 (36). In panel B, the intensity of the background is different from that of panel A because of the difficulty of photographing embryos which do not hybridize to the probe. (C) Enlargement of a stage 37 embryo hybridized with the anti-sense strand RNA probe for XMEK2. 
is distinct from that of XMEK2 (Fig. 7A). While this transcript is detected at all stages examined, the levels of the XMEK 3 transcript show less fluctuation during development than do those of the XMEK2 transcript (Fig. 7A).

Spatial pattern of XMEK2 expression. To determine the pattern of XMEK2 expression during organogenesis, we performed whole-embryo in situ analysis of staged $X$. laevis embryos (Fig. 8A). At stage 17, XMEK2 is initially expressed in the dorsal region of the embryo in a diffuse pattern. Subsequently, expression of XMEK 2 occurs in the early stages of development of the central nervous system, including the brain, spinal cord, and eye. Later (stage 24) expression is found in the hindbrain, midbrain and forebrain, and the somites. By stage 32, expression is detected in the cranial neurons. Expression in the brain is increased, while expression in the spinal cord has decreased by stage 37 (Fig. $8 \mathrm{C})$. No staining is evident in control embryos by using sense RNA (Fig. 8B). This pattern of XMEK2 RNA expression is similar to that of MAP kinase, for which expression is highest in the central nervous system, including the brain, spinal cord, cranial and spinal nerves, and retina (56).

\section{DISCUSSION}

We have identified two cDNA clones (XMEK2 and $\mathrm{XMEK} 3$ ) that code for novel members of the protein kinase family in $X$. laevis. The C-terminal portion of the predicted XMEK2 protein encodes the hallmark amino acid residues and conserved catalytic subdomains $\mathrm{I}$ to $\mathrm{XI}$, which are characteristic of a protein kinase (17). The single exception is a conservative Asp-to-Glu change at residue 195 in subdomain III (Fig. 3). In surveys of the protein kinase family, substitutions have been found at all invariant residues, with the exception of the catalytic lysine in subdomain II (17). The amino acid sequences in domains VI (DIKPSN) and VIII (GCRPYMAPER) suggest that this predicted protein kinase is a member of the serine/threonine kinase subfamily (30). Since the characterized MAP kinases require both tyrosine and threonine phosphorylations for activation, kinases that singly activate MAP kinase must be capable of phosphorylating both types of residues. No definitive motifs that distinguish single specificity protein kinases from dual specificity kinases able to phosphorylate both tyrosine and serine/threonine residues have been identified. However, the presence of a small noncharged amino acid between Pro and Asn in domain VIB (in this clone, the Ser in DIKPSN) loosely correlates with dual specificity (30). The isolation of XMEK 2 cDNAs with insertions of 11 amino acids in the $N$ terminus demonstrates that XMEK2 is subject to alternative processing.

To address the question of whether XMEK2 is also functionally related to other MAP kinase activator proteins, we tested its ability to function in the PKCl-mediated MAP kinase activation pathway of $S$. cerevisiae. In this pathway, MKK1 and MKK2 are redundant MAP-kinase activator proteins that function after the BCK1 kinase and before the MAP kinase homolog MPK1 (Fig. 1) (22, 28, 29). We demonstrated that expression of XMEK2 in yeast cells suppressed the cell lysis defect associated with loss of MKK1 and MKK2. The ability to correct the PKC1 pathway defect was specific for the MKK1 MKK2 step because XMEK2 expression did not suppress loss of MPK1. These results are consistent with XMEK2 providing MAP-kinase activator function.

The limited sequence information for the second cDNA clone (XMEK3) reveals an open reading frame that encodes amino acid residues characteristic of kinase subdomains VI to VIII. While the predicted XMEK2 and XMEK 3 protein kinases are unique, they do share significant identities with the members of the STE7 kinase family $(1,7,22,27,33,51$, $54,55)$. The extent of these identities is within the range of relationships among the previously identified members of this family.

MAPKK, which was previously the sole identified member of this family in amphibians, XMEK2, and XMEK3 share 46 to $63 \%$ identity at the amino acid level (Fig. 2) (27). In $X$. laevis, allelic differences or divergence between duplicate copies of a gene within the tetraploid genome is generally less than $10 \%$ at both the nucleotide and amino acid levels (25). The DNA sequences in subdomains VI to VIII of multiple, independently isolated XMEK 2 cDNA clones representing both XMEK 2 alleles showed $100 \%$ identity at both the nucleotide and amino acid levels to the original stage 17 XMEK2 cDNA clone (data not shown). The similarities between XMEK 2 and XMEK 3 at the nucleotide and the amino acid levels are no greater than the similarities between either of these genes and MAPKK. On the basis of these differences, we conclude that the predicted protein kinases encoded by XMEK 2 and XMEK 3 represent two novel and unique members of the MAP kinase activator family in $X$. laevis.

In amphibians, biochemical studies have identified a single MAP kinase (p42 MAPK) and a single MAP kinase activator (MAPKK). p42 MAPK and MAPKK are expressed in several tissues, including the central nervous system, kidneys, liver, and intestine and the hematopoietic system $(26,56)$. The patterns of XMEK2 expression in $X$. laevis during early embryonic and larval development are similar to those of p42 MAPK and MAPKK $(26,56)$. In the embryo, XMEK2, MAPKK and p42 MAPK are expressed as abundant maternal messages. The patterns of expression of p42 MAPK and MAPKK have not been examined during later embryonic development, and comparisons to XMEK2 expression cannot be made at this time. A murine homolog of XMEK2 (MMEK2) displays a pattern of expression similar to that of XMEK2 (21). MMEK2 is abundantly expressed in adult brain and muscle. Interestingly, XMEK 2 , MAPKK, and p42 MAPK are all expressed in the central nervous system. These results and the demonstrated coexpression of XMEK2 and p42 MAPK in XTC cells suggest that certain cell populations are capable of expressing at least two MAP kinase activators (56).

The pattern of expression of XMEK 3 in embryonic development is distinct from that of XMEK2, p42 MAPK, and MAPKK. Whereas XMEK2 is expressed most abundantly in oocytes and then at markedly lower levels later in development, the levels of XMEK 3 vary less. These unique patterns and the identification of multiple members of the MAP kinase activator family suggest that parallel signaling pathways exist in $X$. laevis.

Precedence for parallel but structurally related signaling pathways exists in $S$. cerevisiae in which conserved units of protein kinases function in three independent pathways. Equivalent tiers in these functionally distinct signaling pathways are composed of MAP kinase and MAP kinase activator family members (11) (Fig. 1). Since each yeast pathway stimulates a different response and requires a unique extracellular stimulus, different stages of embryonic development in $X$. laevis could require activation of distinct MAP kinasedependent signal transduction pathways. Previous studies of $X$. laevis MAP kinase-dependent signal transduction have been confined to oocyte maturation. Analyses of MAP 
kinases in later stages of amphibian development should shed light on whether MAP kinases distinct from p42 MAPK might function with XMEK3. The homologies that we have noted at the structural and functional levels between MAP kinases and MAP kinase activators in $S$. cerevisiae and in vertebrates along with the recent discoveries of multiple family members within both $S$. cerevisiae and $X$. laevis suggest that common reiterated units of protein kinases are utilized for intracellular signal transmission in both systems.

\section{ACKNOWLEDGMENTS}

We are grateful to $\mathrm{K}$. Irie and $\mathrm{K}$. Matsumoto for providing us with the $m k k 1 \Delta m k k 2 \Delta$ strain and the $M K K 1$ plasmids and sequence prior to publication, to D. E. Levin for the MPK1 plasmid, and to G. Boguslawski (Indiana University Medical Center) for the GBH38 strain. We thank O. Piedad for technical help and R. K. Esch, D. E. Levin, and $\mathrm{K}$. Bloom for helpful comments on the manuscript.

This work was supported by U.S. Public Health Service grant GM-39852 to B.E., grant HL-32262 to L.I.Z., and a Markey fellowship to C.K.

\section{REFERENCES}

1. Boguslawski, G., and J. O. Polazzi. 1987. Complete nucleotide sequence of a gene conferring polymyxin B resistance on yeast: similarity of the predicted polypeptide to protein kinases. Proc. Natl. Acad. Sci. USA 84:5848-5852.

2. Brewster, J. L., T. de Valoir, N. D. Dwyer, E. Winter, and M. C. Gustin. 1993. An osmo-sensing signal transduction pathway in yeast. Science 259:1760-1763.

3. Cairns, B. R., S. W. Ramer, and R. D. Kornberg. 1992. Order of action of components in the yeast pheromone response pathway revealed with a dominant allele of the STE11 kinase and the multiple phosphorylation of the STE7 kinase. Genes Dev. 6:1305-1318.

4. Chomczynski, P., and N. Sacchi. 1987. Single-step method of RNA isolation by acid guanidinium thiocyanate-phenol-chloroform extraction. Anal. Biochem. 162:156-159.

5. Cobb, M. H., T. G. Boulton, and D. J. Robbins. 1991. Extracellular signal-regulated kinases: ERKs in progress. Cell Regul. 2:965-978.

6. Courchesne, W. E., R. Kunisawa, and J. Thorner. 1989. A putative protein kinase overcomes pheromone-induced arrest of cell cycling in $S$. cerevisiae. Cell 58:1107-1119.

7. Crews, C. M., A. Alessandrini, and R. L. Erickson. 1992. The primary structure of MEK, a protein kinase that phosphorylates the ERK gene product. Science 258:478-480.

8. Crews, C. M., and R. L. Erickson. 1992. Purification of a murine protein-tyrosine/threonine kinase that phosphorylates and activates the $E R K-1$ gene product: relationship to the fission yeast byrl gene product. Proc. Natl. Acad. Sci. USA 89:8205-8209.

9. Elion, E. A., P. L. Grisafi, and G. R. Fink. 1990. FUS3 encodes a cdc2+/CDC28-related kinase required for the transition from mitosis into conjugation. Cell 60:649-664.

10. Errede, B., A. Gartner, Z. Zhou, K. Nasmyth, and G. Ammerer. 1993. A pheromone induced kinase cascade in $S$. cerevisiae: activation of the FUS3 kinase by the STE7 kinase in vitro. Nature (London) 362:261-264.

11. Errede, B., and D. E. Levin. 1993. A conserved kinase cascade for MAP kinase activation in yeast. Curr. Opin. Cell Biol. 5:254-260.

12. Ferrell, J. E., Jr., M. Wu, J. C. Gerhart, and G. S. Martin. 1991 Cell cycle tyrosine phosphorylation of $\mathrm{p} 34^{\mathrm{cdc} 2}$ and a microtubule-associated protein kinase homolog in Xenopus oocytes and eggs. Mol. Cell. Biol. 11:1965-1971.

13. Gartner, A., K. Nasmyth, and G. Ammerer. 1992. Signal transduction in Saccharomyces cerevisiae requires tyrosine and threonine phosphorylation of FUS3 and KSS1. Genes Dev. 6:1280-1292.

14. Genetics Computer Group. 1991. Program manual for the GCG package, version 7. Genetics Computer Group, Madison, Wis.

15. Gietz, R. D., and A. Sugino. 1988. New yeast-Escherichia coli shuttle vectors constructed with in vitro mutagenized yeast genes lacking six-base pair restriction sites. Gene 74:527-534.

16. Gotoh, Y., K. Moriyama, S. Matsuda, E. Okumura, T. Kishimoto, H. Kawasaki, K. Suzuki, I. Yahara, H. Sakai, and E. Nishida. 1991. Xenopus M phase MAP kinase: isolation of its cDNA and activation by MPF. EMBO J. 10:2661-2668.

17. Hanks, S. K., and A. M. Quinn. 1991. Protein kinase catalytic domain sequence database: identification of conserved features of primary structure and classification of family members. Methods Enzymol. 200:38-62.

18. Hemmati-Brivanlou, A., D. Frank, M. E. Bolce, B. D. Brown, H. L. Sive, and R. M. Harland. 1990. Localization of specific mRNAs in Xenopus embryos by whole-mount in situ hybridization. Development 110:325-330.

19. Henikof, S. 1987. Unidirectional digestion with exonuclease III in DNA sequence analysis. Methods Enzymol. 155:156-165.

20. Ho, S. N., H. D. Hunt, R. M. Horton, J. K. Pullen, and L. R. Pease. 1989. Site-directed mutagenesis by overlap extension using polymerase chain reaction. Gene 77:51-59.

21. Hughes, R., and L. Zon (Children's Hospital). 1993. Personal communication.

22. Irie, K., M. Takase, K. S. Lee, D. E. Levin, H. Araki, K. Matsumoto, and Y. Oshima. 1993. MKK1 and MKK2, which encode Saccharomyces cerevisiae mitogen-activated protein kinase-kinase homologs, function in the pathway mediated by protein kinase C. Mol. Cell. Biol. 13:3076-3083.

23. Issacs, H. V., D. Tannahill, and J. M. W. Slack. 1992. Expression of a novel EGF in Xenopus embryo. A new candidate inducing factor for mesoderm formation and anteroposterior specification. Development 114:711-720.

24. Ito, H., Y. Fukuda, K. Murata, and A. Kimura. 1983. Transformation of intact yeast cells treated with alkali cations. J. Bacteriol. 153:163-168.

25. Knochel, W., E. Korge, A. Basner, and W. Meyerhof. 1986. Globin evolution in the genus Xenopus: comparative analysis of cDNAs coding for adult globin polypeptides of Xenopus borealis and Xenopus tropicalis. J. Mol. Evol. 23:211-223.

26. Kosako, H., Y. Gotoh, S. Matsuda, M. Ishikawa, and E. Nishida. 1992. Xenopus MAP kinase activator is a serine/threonine/ tyrosine kinase activated by threonine phosphorylation. EMBO J. 11:2903-2908.

27. Kosako, H., E. Nishida, and Y. Gotoh. 1993. cDNA cloning of MAP kinase kinase reveals cascade pathways in yeast to vertebrates. EMBO J. 12:787-794.

28. Lee, K. S., K. Irie, Y. Gotoh, Y. Watanabe, H. Araki, E. Nishida, K. Matsumoto, and D. E. Levin. 1993. A yeast mitogenactivated protein kinase homolog (MPK1p) mediates signalling by protein kinase C. Mol. Cell. Biol. 13:3067-3075.

29. Lee, K. S., and D. E. Levin. 1992. Dominant mutations in a gene encoding a putative protein kinase (BCK1) bypass the requirement for a Saccharomyces cerevisiae protein kinase $\mathrm{C}$ homolog. Mol. Cell. Biol. 12:172-182.

30. Lindberg, R. A., A. M. Quinn, and T. Hunter. 1992. Dualspecificity protein kinases: will any hydroxyl do? Trends Biochem. Sci. 17:114-119.

31. Marsh, L., A. Neiman, and I. Herskowitz. 1991. Signal transduction during pheromone response in yeast. Annu. Rev. Cell Biol. 7:699-728.

32. Matsuda, S., H. Kosako, K. Takenaka, K. Moriyama, H. Sakai, T. Akiyama, Y. Gotoh, and E. Nishida. 1992. Xenopus MAP kinase activator: identification and function as a key intermediate in the phosphorylation cascade. EMBO J. 11:973-982.

33. Nadin-Davis, S., and A. Nasim. 1988. A gene which encodes a predicted protein kinase can restore some functions of the ras gene in fission yeast. EMBO J. 7:985-993.

34. Nakielny, S., D. G. Campbell, and P. Cohen. 1992. MAP kinase kinase from rabbit skeletal muscle. A novel dual specificity enzyme showing homology to yeast protein kinases involved in pheromone-dependent signal transduction. FEBS Lett. 308: 183-189.

35. Nakielny, S., P. Cohen, J. Wu, and T. Sturgill. 1992. MAP kinase activator from insulin-stimulated skeletal muscle is a protein threonine/tyrosine kinase. EMBO J. 11:2123-2129. 
36. Nieuwkoop, P. D., and J. Faber. 1967. Normal table of Xenopus laevis (Daudin), 2nd ed. North Holland Publishing Co., Amsterdam.

37. Pearson, W., and D. Lipman. 1988. Improved tools for biological sequencing. Proc. Natl. Acad. Sci. USA 85:2444-2448.

38. Pelech, S. L., and J. S. Sanghera. 1992. Mitogen-activated protein kinases: versatile transducers for cell signaling. Trends Biochem. Sci. 17:233-238.

39. Posada, J., and J. A. Cooper. 1992. Requirements for phosphorylation of MAP kinase during meiosis in Xenopus oocytes. Science 255:212-215.

40. Posada, J., J. Sanghera, S. Pelech, R. Aebersold, and J. A. Cooper. 1991. Tyrosine phosphorylation and activation of homologous protein kinases during oocyte maturation and mitogenic activation of fibroblasts. Mol. Cell. Biol. 11:2517-2528.

41. Rhodes, N., M. Company, and B. Errede. 1990. A yeastEscherichia coli shuttle vector containing the M13 origin of replication. Plasmid 23:159-162.

42. Rhodes, N., L. Connell, and B. Errede. 1990. STE11 is a protein kinase required for cell-type-specific transcription and signal transduction in yeast. Genes Dev. 4:1862-1874.

43. Saiki, R. K., D. H. Gelfand, S. Stoffel, S. J. Scharf, R. Higuchi, G. T. Horn, K. B. Mullis, and H. A. Erlich. 1988. Primerdirected enzymatic amplification of DNA with a thermostable DNA polymerase. Science 239:487-491.

44. Sambrook, J., E. F. Fritsch, and T. Maniatis. 1989. Molecular cloning: a laboratory manual, 2nd ed. Cold Spring Harbor Laboratory Press, Cold Spring Harbor, N.Y.

45. Sanger, F., S. Nicklen, and A. R. Coulson. 1977. DNA sequencing with chain-terminating inhibitors. Proc. Natl. Acad. Sci. USA 74:5463-5467.

46. Sherman, F., G. R. Fink, and J. B. Hicks. 1979. Methods in yeast genetics. Cold Spring Harbor Laboratory, Cold Spring Harbor, N.Y.

47. Sprague, G. F., Jr. 1991. Assays of yeast mating reactions. Methods Enzymol. 194:77-93.
48. Sprague, G. F., Jr. 1991. Signal transduction in yeast mating: receptors, transcription factors, and the kinase connection. Trends Genet. 7:393-398.

49. Staden, R. 1982. Automation of the computer handling of gel reading data produced by the shotgun method of DNA sequencing. Nucleic Acids Res. 10:4731-4751.

50. Stevenson, B. J., N. Rhodes, B. Errede, and G. F. Sprague, Jr. 1992. Constitutive mutants of the protein kinase STE11 activate the yeast pheromone response pathway in the absence of the $G$ protein. Genes Dev. 6:1293-1304.

51. Teague, M. A., D. T. Chaleff, and B. Errede. 1986. Nucleotide sequence of the yeast regulatory gene STE7 predicts a protein homologous to protein kinases. Proc. Natl. Acad. Sci. USA 83:7371-7375.

52. Thomas, G. 1992. MAP kinase by any other name smells just as sweet. Cell 68:3-6.

53. Thomsen, G., T. Woolf, M. Whitman, S. Sokol, J. Vaughan, W. Vale, and D. A. Melton. 1990. Activins are expressed early in Xenopus embryogenesis and can induce axial mesoderm and anterior structures. Cell 63:485-493.

54. Warbrick, E., and P. A. Fantes. 1991. The wis1 protein kinase is a dosage-dependent regulator of mitosis in Schizosaccharomyces pombe. EMBO J. 10:4291-4299.

55. Wu, J., J. K. Harrison, L. A. Vincent, C. Haystead, T. A. J. Haystead, H. Michel, D. F. Hunt, K. R. Lynch, and T. W. Sturgill. 1993. Molecular structure of a protein-tyrosine/threonine kinase activating p42 mitogen-activated protein (MAP) kinase: MAP kinase kinase. Proc. Natl. Acad. Sci. USA 90:173177.

56. Zaitsevskaya, T., and J. A. Cooper. 1993. Developmentallyregulated expression of a mitogen-activated protein (MAP) kinase in Xenopus laevis. Cell Growth Differ. 3:773-782.

57. Zhou, Z., A. Gartner, R. Cade, G. Ammerer, and B. Errede. 1993. Pheromone induced signal transduction in Saccharomyces cerevisiae requires the sequential function of three protein kinases. Mol. Cell. Biol. 13:2069-2080. 